EPJ Web of Conferences 70, 00031 (2014)

DOI: $10.1051 /$ epjconf/ 20147000031

(C) Owned by the authors, published by EDP Sciences, 2014

\title{
Hard-Thermal-Loop QCD thermodynamics and quark number susceptibility
}

\author{
Sylvain Mogliacci ${ }^{1}$ and Nan Su${ }^{1, a}$ \\ ${ }^{1}$ Fakultät für Physik, Universität Bielefeld, D-33615 Bielefeld, Germany
}

\begin{abstract}
The weak-coupling expansion of the QCD pressure is known up to the order $g^{6} \log g$. However, at experimentally relevant temperatures, the corresponding series is poorly convergent. In this proceedings, we discuss at which extent the gauge-invariant resummation scheme, Hard-Thermal-Loop perturbation theory (HTLpt), improves the apparent convergence. We first present HTLpt results for QCD thermodynamic functions up to three-loop order at vanishing chemical potential. Then, we report a preliminary HTLpt result of one-loop quark number susceptibility, probing the finite density equation of state. Our results are consistent with lattice data down to $2-3 T_{c}$, reinforcing the weakly-coupled quasiparticle picture in the intermediate coupling regime.
\end{abstract}

\section{Introduction}

The heavy-ion experiments that are currently running start at temperatures on the order of $T \sim$ $350 \mathrm{MeV}$ for RHIC and $T \sim 600-800 \mathrm{MeV}$ for the LHC. In this range of temperature, the use of conventional perturbation theory does not guaranty the convergence of the quantity under study, since the coupling is neither infinitesimally weak, nor infinitely strong with $g \sim 2$, corresponding to $\alpha_{S} \sim 0.3$. Indeed, the perturbative evaluation of the pressure, which has a long history [1], turned out to show a problem of apparent convergence, regarding the successive approximations to various thermodynamic quantities. Interestingly enough, this problem persists even at very high temperatures $\left(T \sim 10^{5} \mathrm{GeV}\right)$. It was shown that because of the soft contribution for which the typical momentum in a thermal medium is of order $\sim g T$, the total series get spoiled by a contribution in power of $g$. A more detailled investigation on this problem can be found in Ref. [2]. As an illustration of this, we refer to Fig. 1 which displays the successive weak-coupling expansion series of the pressure up to the order $g^{5}$. The bands are obtained by varying the renormalization scale $\Lambda$ by a factor of 2 around the value $\Lambda=2 \pi T$ and we use three-loop running for $\alpha_{s}\left(=g^{2} / 4 \pi\right)$ [3] with $\Lambda_{\overline{\mathrm{MS}}}\left(N_{f}=3\right)=344 \mathrm{MeV}$ [4]. The oscillating behavior of the perturbative series calls the need of reorganizing the expansion, in order to be able to use the machinery of perturbation theory in practice to give quantitative predictions for the ongoing experiments.

In this proceedings, we address the state-of-the-art in the reorganization of the thermal QCD perturbative series, using the framework of Hard-Thermal-Loop perturbation theory (HTLpt) which amounts to expand around a gas of non-interacting massive quasiparticles, therefore accounting for the main effects in a quark gluon plasma such as Debye screening, quasiparticles, and Landau damping.

\footnotetext{
${ }^{\mathrm{a}}$ Speaker
} 


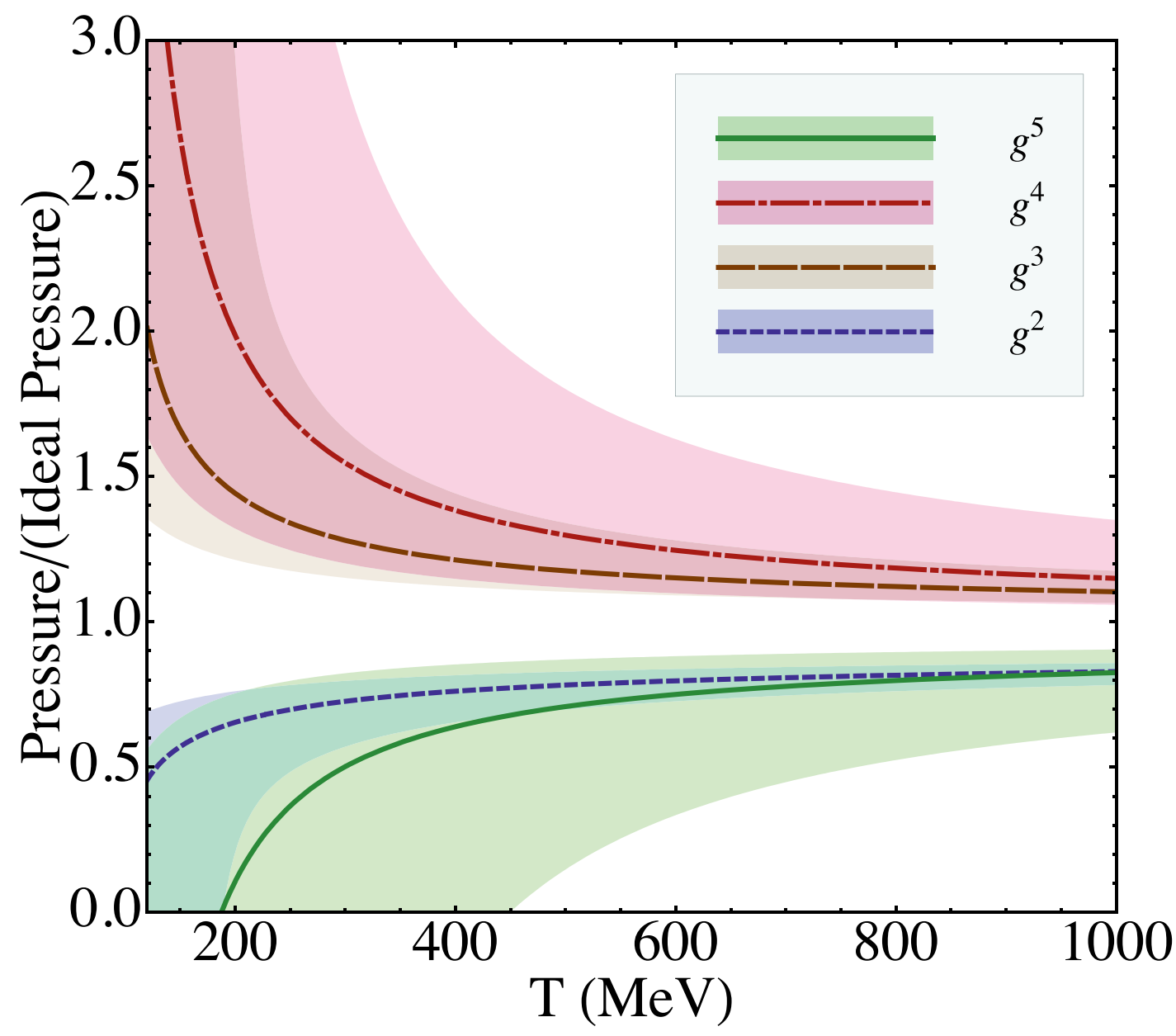

Figure 1. Weak-coupling expansion for the scaled QCD pressure with $N_{f}=3$. Shaded bands show the result of varying the renormalization scale $\Lambda$ by a factor of 2 around $\Lambda=2 \pi T$.

In the following, we first briefly introduce the setup of HTLpt. Then, we discuss the three-loop thermodynamic functions at vanishing chemical potential, and present a preliminary result on a recent HTLpt computation of the leading order quark number susceptibility. Indeed, this type of quantities is well known to be of great importance, since it directly probes the response of a dense medium to the fluctuation of quark densities, while being able to be computed on the lattice. In the following, all our results are subsequently compared to lattice data.

\section{Hard-Thermal-Loop perturbation theory}

Hard-Thermal-Loop perturbation theory is a self-consistent, systematic and gauge-invariant way of reorganizing the finite temperature perturbative series of QCD. It is a generalization of Screened Perturbation Theory (SPT) [5] to gauge theory, and it implements order by order in perturbation theory HTL dressed propagators and vertices. Formally, one adds and subtracts a term in the Lagrangian 
density of QCD, treating the added term in the free part of the Lagrangian, while implementing the subtracted one as an interaction. In order to preserve gauge invariance, this term can no longer be a local mass term as in SPT, and one uses the following density Lagrangian:

$$
\mathcal{L}=\left.\left(\mathcal{L}_{\mathrm{QCD}}+\mathcal{L}_{\mathrm{HTL}}\right)\right|_{g \rightarrow \sqrt{\delta} g}+\Delta \mathcal{L}_{\mathrm{HTL}}
$$

where $\Delta \mathcal{L}_{\mathrm{HTL}}$ contains the counter terms related to the new part $\mathcal{L}_{\mathrm{HTL}}$, which is nothing but the HTL effective Lagrangian, that is the infinitely high temperature limit of the QCD Lagrangian. Let us remind that this term reads:

$$
\begin{aligned}
\mathcal{L}_{\mathrm{HTL}}= & -\frac{1}{2}(1-\delta) m_{D}^{2} \operatorname{Tr}\left(F_{\mu \alpha}\left\langle\frac{y^{\alpha} y^{\beta}}{(y \cdot D)^{2}}\right\rangle_{y} F_{\beta}^{\mu}\right) \\
& +(1-\delta) i m_{q}^{2} \bar{\psi} \gamma^{\mu}\left\langle\frac{y_{\mu}}{y \cdot D}\right\rangle_{y} \psi,
\end{aligned}
$$

where $y^{\mu}$ is a lightlike 4-vector, $m_{D}$ and $m_{q}$ are the Debye screening mass and thermal quark mass parameters, and $\delta$ is the expansion parameter amounting for the number of HTL dressed loops.

Physical observables are calculated in HTLpt by expanding them in powers of $\delta$, truncating at some specified order, and then setting $\delta=1$. This defines a reorganization of the perturbation series in which the effects of $m_{D}^{2}$ and $m_{q}^{2}$ terms in (2) are included to all orders but then systematically subtracted out at higher orders in perturbation theory. If we set $\delta=1$, the HTLpt Lagrangian (1) reduces to the QCD Lagrangian. If the expansion in $\delta$ could be calculated to all orders the final result would not depend on $m_{D}$ and $m_{q}$ when we set $\delta=1$. However, any truncation of the expansion in $\delta$ produces results that depend on $m_{D}$ and $m_{q}$. Some prescription is required to determine $m_{D}$ and $m_{q}$ as a function of $T$ and $\alpha_{s}$.

In the following, we would like to make notice that for convenience, all dimensionful parameters will be rescaled to be dimensionless as $\hat{x}_{D} \equiv \frac{x}{2 \pi T}$. In addition, the free energy of non-interacting gluons will be denoted as:

$$
\mathcal{F}_{\text {free }}=-\left(N_{c}^{2}-1\right) \frac{\pi^{2} T^{4}}{45}
$$

With the standard normalization, we have $c_{A}=N_{c}, d_{A}=N_{c}^{2}-1, s_{F}=N_{f} / 2, d_{F}=N_{c} N_{f}$, and $s_{2 F}=\left(N_{c}^{2}-1\right) N_{f} / 4 N_{c}$. 


\section{NNLO thermodynamics at vanishing $\mu$}

\subsection{Thermodynamic potential}

At three-loop order, aka next-to-next-to-leading order (NNLO), and vanishing chemical potential $\mu$, one obtains [6] for QCD with $N_{c}$ colors and $N_{f}$ flavors, the following thermodynamic potential:

$$
\begin{aligned}
& \frac{\Omega_{\mathrm{NNLO}}^{T}}{\mathcal{F}_{\text {free }}}=1+\frac{7}{4} \frac{d_{F}}{d_{A}}-\frac{15}{4} \hat{m}_{D}^{3}+\frac{c_{A} \alpha_{s}}{3 \pi}\left[-\frac{15}{4}+\frac{45}{2} \hat{m}_{D}\right. \\
& \left.-\frac{135}{2} \hat{m}_{D}^{2}-\frac{495}{4}\left(\log \frac{\hat{\Lambda}}{2}+\frac{5}{22}+\gamma_{E}\right) \hat{m}_{D}^{3}\right] \\
& +\frac{s_{F} \alpha_{s}}{\pi}\left[-\frac{25}{8}+\frac{15}{2} \hat{m}_{D}+15\left(\log \frac{\hat{\Lambda}}{2}-\frac{1}{2}+\gamma_{E}\right.\right. \\
& \left.+2 \log 2) \hat{m}_{D}^{3}-90 \hat{m}_{q}^{2} \hat{m}_{D}\right]+\left(\frac{c_{A} \alpha_{s}}{3 \pi}\right)^{2}\left[\frac{45}{4} \frac{1}{\hat{m}_{D}}\right. \\
& -\frac{165}{8}\left(\log \frac{\hat{\Lambda}}{2}-\frac{84}{55}-\frac{72}{11} \log \hat{m}_{D}-\frac{6}{11} \gamma_{E}\right. \\
& \left.-\frac{74}{11} \frac{\zeta^{\prime}(-1)}{\zeta(-1)}+\frac{19}{11} \frac{\zeta^{\prime}(-3)}{\zeta(-3)}\right)+\frac{1485}{4}\left(\log \frac{\hat{\Lambda}}{2}-\frac{79}{44}\right. \\
& \left.\left.+\gamma_{E}+\log 2-\frac{\pi^{2}}{11}\right) \hat{m}_{D}\right]+\left(\frac{c_{A} \alpha_{s}}{3 \pi}\right)\left(\frac{s_{F} \alpha_{s}}{\pi}\right)\left[\frac{15}{2} \frac{1}{\hat{m}_{D}}\right. \\
& -\frac{235}{16}\left(\log \frac{\hat{\Lambda}}{2}-\frac{144}{47} \log \hat{m}_{D}-\frac{24}{47} \gamma_{E}+\frac{319}{940}\right. \\
& \left.+\frac{111}{235} \log 2-\frac{74}{47} \frac{\zeta^{\prime}(-1)}{\zeta(-1)}+\frac{1}{47} \frac{\zeta^{\prime}(-3)}{\zeta(-3)}\right)+\frac{315}{4} \\
& \left.\left(\log \frac{\hat{\Lambda}}{2}-\frac{8}{7} \log 2+\gamma_{E}+\frac{9}{14}\right) \hat{m}_{D}+90 \frac{\hat{m}_{q}^{2}}{\hat{m}_{D}}\right] \\
& +\left(\frac{s_{F} \alpha_{s}}{\pi}\right)^{2}\left[\frac{5}{4} \frac{1}{\hat{m}_{D}}+\frac{25}{12}\left(\log \frac{\hat{\Lambda}}{2}+\frac{1}{20}+\frac{3}{5} \gamma_{E}\right.\right. \\
& \left.-\frac{66}{25} \log 2+\frac{4}{5} \frac{\zeta^{\prime}(-1)}{\zeta(-1)}-\frac{2}{5} \frac{\zeta^{\prime}(-3)}{\zeta(-3)}\right)-15\left(\log \frac{\hat{\Lambda}}{2}\right. \\
& \left.\left.-\frac{1}{2}+\gamma_{E}+2 \log 2\right) \hat{m}_{D}+30 \frac{\hat{m}_{q}^{2}}{\hat{m}_{D}}\right]+s_{2 F}\left(\frac{\alpha_{s}}{\pi}\right)^{2} \\
& {\left[\frac{15}{64}(35-32 \log 2)-\frac{45}{2} \hat{m}_{D}\right]+O\left(\hat{m}_{D}^{6}, \hat{m}_{q}^{6}\right),}
\end{aligned}
$$

which is obtained using dimensional regularization in the $\overline{\mathrm{MS}}$ scheme with $\Lambda$ the renormalization scale.

\subsection{Mass prescriptions}

In order to get free energy from the thermodynamic potential, some prescriptions need to be imposed to determine the mass parameters in terms of the original parameters of QCD. It is important that one should make the choice of the mass parameters according to physical motivations. One natural choice would be to impose the Principle of Minimum Sensitivity (PMS), which requires the computed 
quantity depends the least on the parameters. Unfortunately, it has been shown [6] that at NNLO, the PMS gives a complex value for $m_{D}$. In addition, the weak-coupling expansion for $m_{D}$ beyond leading order (LO) involves the nonperturbative magnetic scale $\sim g^{2} T$, which breaks down perturbation theory by introducing infrared divergences. Therefore, at this order, a fairly physically motivated choice would be to chose the next-to-leading order (NLO) electric mass computed from dimensional reduction which is well defined to all orders in perturbation theory [7], as it was done in [6]. Attributing this value to our Debye mass parameter $m_{D}$, we have:

$$
\begin{aligned}
m_{D}^{2}= & \frac{4 \pi \alpha_{s}}{3} T^{2}\left[c_{A}+s_{F}+\frac{c_{A}^{2} \alpha_{s}}{3 \pi}\left(\frac{5}{4}+\frac{11}{2} \gamma_{E}+\frac{11}{2} \log \frac{\hat{\Lambda}}{2}\right)\right. \\
& +\frac{c_{A} s_{F} \alpha_{s}}{\pi}\left(\frac{3}{4}-\frac{4}{3} \log 2+\frac{7}{6} \gamma_{E}+\frac{7}{6} \log \frac{\hat{\Lambda}}{2}\right) \\
& \left.+\frac{s_{F}^{2} \alpha_{s}}{\pi}\left(\frac{1}{3}-\frac{4}{3} \log 2-\frac{2}{3} \gamma_{E}-\frac{2}{3} \log \frac{\hat{\Lambda}}{2}\right)-\frac{3}{2} \frac{s_{2 F} \alpha_{s}}{\pi}\right] .
\end{aligned}
$$

The thermal quark mass is chosen to be the solution from the NNLO PMS gap equation, and gives $m_{q}=0$. Notice that the quark contribution to the total free energy is still present, thanks to the mixed quark and gluon contributions.

\subsection{Thermodynamic functions}

In this section, we present NNLO HTLpt results for pressure and trace anomaly at vanishing chemical potential. We will focus on $N_{f}=3 \mathrm{QCD}$. For the strong coupling constant $\alpha_{s}$, we use three-loop running [3] with $\Lambda_{\overline{\mathrm{MS}}}=344 \mathrm{MeV}$ which for $N_{f}=3$ gives $\alpha_{s}(5 \mathrm{GeV})=0.2034$ [4].

\subsubsection{Pressure}

In Fig. 2, we show the LO, NLO, and NNLO HTLpt predictions for the pressure of QCD with $N_{f}=3$ normalized to that of an ideal gas as a function of $T$. The band is again obtained by varying the renormalization scale by a factor of 2 around the central value $\Lambda=2 \pi T$. The $N_{f}=2+1$ lattice data from the Wuppertal-Budapest collaboration use the stout action. Since their results show essentially no dependence on the lattice spacing (it is smaller than the statistical errors), they provide a continuum estimate by averaging the trace anomaly measured using their two smallest lattice spacings corresponding to $N_{\tau}=8$ and $N_{\tau}=10$ [8], which were essentially on top of the $N_{\tau}=6$ measurement [9]. ${ }^{1}$ Using standard lattice techniques, the continuum-estimated pressure is computed from an integral of the trace anomaly. The $N_{f}=2+1$ lattice data from the hotQCD collaboration are their $N_{\tau}=8$ results using both the asqtad and p4 actions [10]. The hotQCD results have not been continuum extrapolated and the error bars correspond to only statistical errors and do not factor in the systematic error associated with the calculation which, for the pressure, is estimated by the hotQCD collaboration to be between $5-10 \%$. As can be seen from Fig. 2, the successive HTLpt approximations represent an improvement over the successive approximations coming from a weak-coupling expansion; however, the NNLO result represents a significant correction to the LO and NLO results. That being said, the

\footnotetext{
${ }^{1}$ It is noted that the Wuppertal-Budapest group has published a few data points for the trace anomaly with $N_{\tau}=12$ and within statistical error bars these are consistent with the published continuum estimated results.
} 
NNLO HTLpt result agrees quite well with the available lattice data down to temperatures on the order of $T \sim 350 \mathrm{MeV}$ for QCD with $N_{f}=3$. $^{2}$

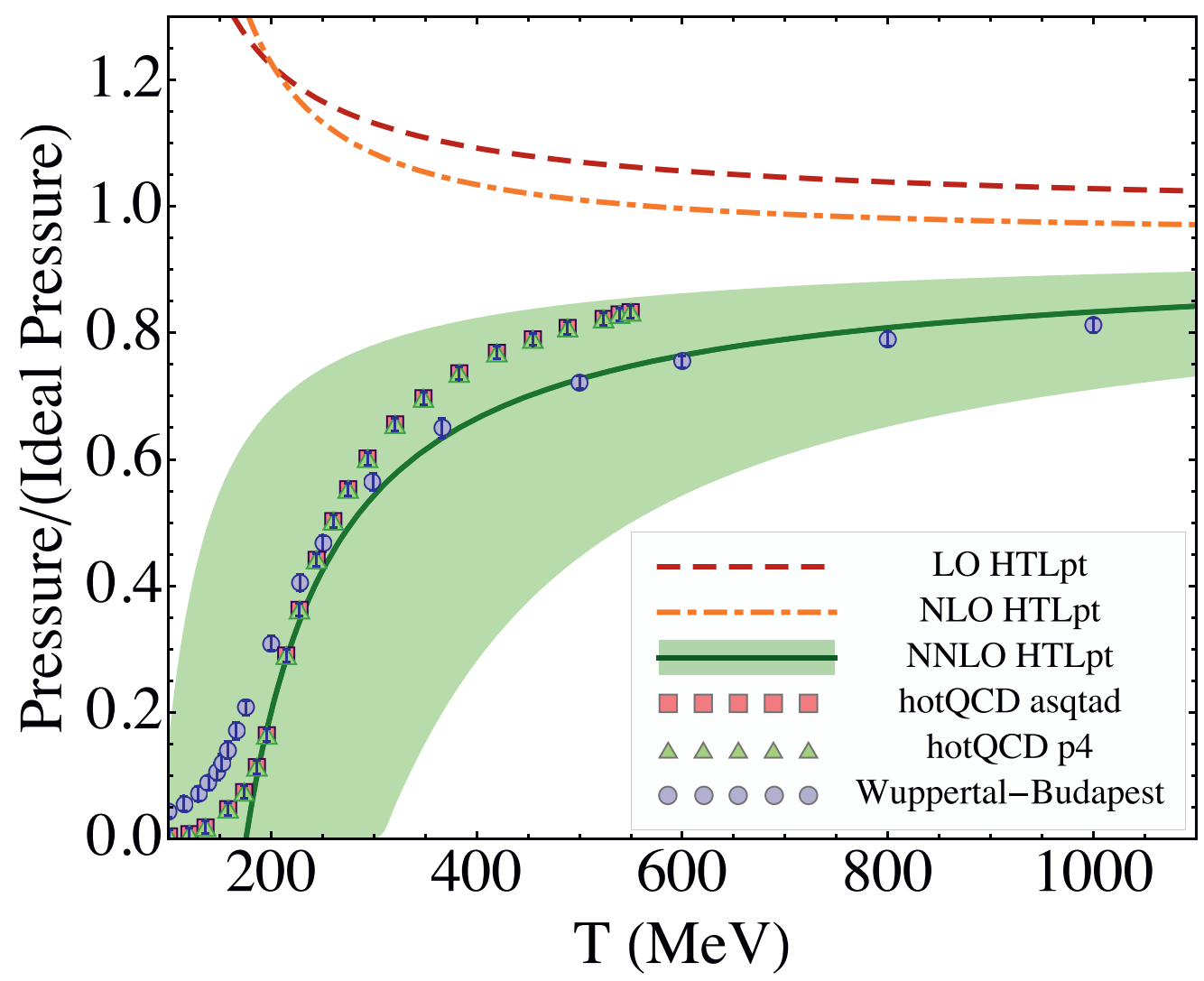

Figure 2. Comparison of LO, NLO, and NNLO predictions for the scaled pressure of $N_{f}=3$ QCD with $N_{f}=2+1$ lattice data from the hotQCD [10] and Wuppertal-Budapest [8] collaborations. Shaded band shows the result of varying the renormalization scale $\Lambda$ by a factor of 2 around $\Lambda=2 \pi T$ for the NNLO result.

\subsubsection{Trace anomaly}

In Fig. 3 we show the NNLO HTLpt prediction for the trace anomaly of QCD with $N_{f}=3$ normalized to $T^{4}$ as a function of $T$. The data from both the Wuppertal-Budapest collaboration and the hotQCD collaboration are taken from the same data sets displayed in Fig. 2 and described previously. In the case of the hotQCD results we note that the results for the trace anomaly using the p4 action show large lattice size affects at all temperatures shown and the asqtad results for the trace anomaly show

\footnotetext{
${ }^{2}$ The Wuppertal-Budapest and hotQCD data were obtained using a physical strange quark mass; however, HTLpt calculations use massless quarks. The difference between massive and massless quarks is expected to be significant only for $T \lesssim 32 \mathrm{MeV}$ corresponding to the temperature where the lowest fermionic Matsubara mode equals the strange quark mass.
} 
large lattice size effects for $T \gtrsim 200 \mathrm{MeV}$. We see very good agreement between the HTLpt prediction and the available lattice data down to temperatures on the order of $T \sim 350 \mathrm{MeV}$.

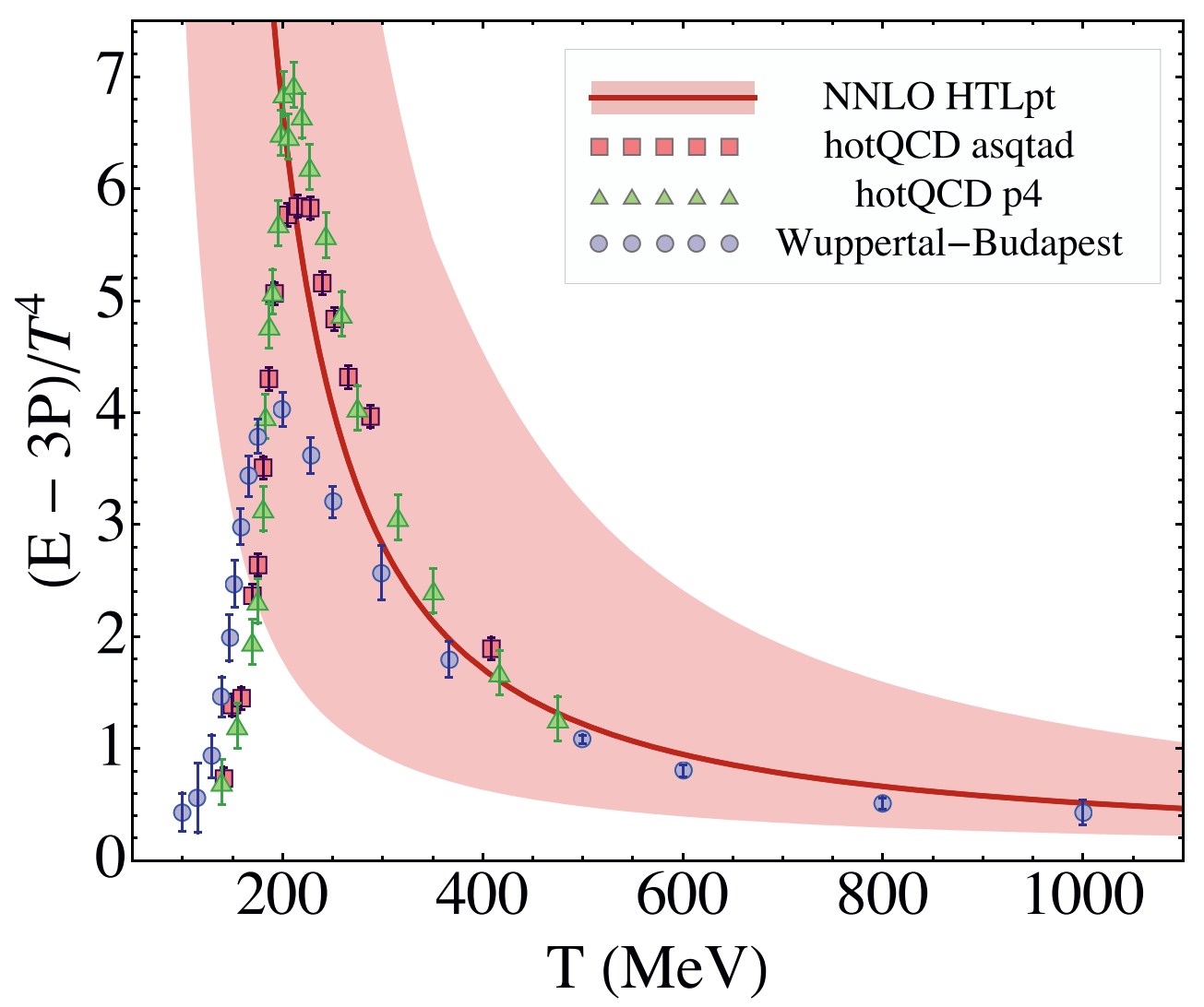

Figure 3. Comparison of LO, NLO, and NNLO predictions for the scaled trace anomaly of $N_{f}=3$ QCD with $N_{f}=2+1$ lattice data from the hotQCD [10] and Wuppertal-Budapest [8] collaborations. Shaded band shows the result of varying the renormalization scale $\Lambda$ by a factor of 2 around $\Lambda=2 \pi T$ for the NNLO result.

\section{LO quark number susceptibility}

\subsection{Introduction}

For simplicity, we assume in the following that all the quarks have degenerate chemical potentials. Then, the relation between the thermodynamic potential $\Omega$ and the diagonal quark number susceptibility $\chi_{\text {uи }}$ reads:

$$
\chi_{u и}(T) \equiv-\left.\frac{\partial^{2} \Omega(T, \mu)}{\partial \mu^{2}}\right|_{\mu=0} .
$$


Due to the massless approximation for quarks in HTLpt, baryon number susceptibility, at the considered order of truncation (see in the following for further explanations), is related to the above quantity by a simple rescaling:

$$
\chi_{B}=\frac{\chi_{u u}}{3} .
$$

In addition, we would like to make notice that the introduction of a non-vanishing chemical potential $\mu$ in the derivation of the thermodynamic potential amounts to the following substitution for Matsubara frequencies in the imaginary-time formalism:

$$
\omega_{n} \rightarrow \omega_{n}-i \mu
$$

\subsection{LO thermodynamic potential at finite $T$ and $\mu$}

As a preliminary result, we give here the high temperature truncation of the full LO thermodynamic potential at finite $T$ and $\mu$. Up to the truncation order $O\left(\hat{m}^{5}\right)$, it reads:

$$
\begin{aligned}
\frac{\Omega_{\mathrm{LO}}^{T, \mu}}{\mathcal{F}_{\text {free }}}= & 1+\frac{d_{F}}{d_{A}}\left(\frac{7}{4}+30 \hat{\mu}^{2}+60 \hat{\mu}^{4}\right) \\
& -\frac{15}{2} \hat{m}_{D}^{2}-30 \frac{d_{F}}{d_{A}}\left(1+12 \hat{\mu}^{2}\right) \hat{m}_{q}^{2} \\
& +30 \hat{m}_{D}^{3}+60 \frac{d_{F}}{d_{A}}\left(6-\pi^{2}\right) \hat{m}_{q}^{4} \\
& +\frac{45}{4}\left(\gamma_{E}-\frac{7}{2}+\frac{\pi^{2}}{3}+\log \frac{\hat{\Lambda}}{2}\right) \hat{m}_{D}^{4} \\
& +O\left(\hat{m}_{D}^{6}, \hat{m}_{q}^{6}\right) .
\end{aligned}
$$

\subsection{Mass prescriptions}

The LO gap equation from PMS is not well defined in HTLpt, we therefore identify $m_{D}$ and $m_{q}$ by their weak-coupling values

$$
\begin{aligned}
& m_{D}^{2}=\alpha_{S} \pi\left[\frac{4}{3}\left(N_{c}+\frac{1}{2} N_{f}\right) T^{2}+2 N_{f} \frac{\mu^{2}}{\pi^{2}}\right], \\
& m_{q}^{2}=\alpha_{S} \pi \frac{N_{c}^{2}-1}{4 N_{c}}\left(T^{2}+\frac{\mu^{2}}{\pi^{2}}\right) .
\end{aligned}
$$

Note that the gluons also carry $\mu$ dependence, hence contribute to the final result.

\subsection{Result}

In Fig. 4 we show the LO baryon number susceptibility for $N_{f}=3$ QCD normalized by $T^{2}$ as a function of $T$ [11]. We use one-loop running with $\Lambda_{\overline{\mathrm{MS}}}=176 \mathrm{MeV}$ which is derived from a recent lattice measurement of the strong coupling constant at $1.5 \mathrm{GeV}$ [12]. The band is again obtained by varying the renormalization scale by a factor of 2 around the central value $\Lambda=2 \pi T$. Lattice data from both the HotQCD [13] and Wuppertal-Budapest [14] collaborations are continuum extrapolated. From the comparison, we see that the LO result does quite a reasonable job and is consistent with the lattice data down to $T \sim 250 \mathrm{MeV}$. 


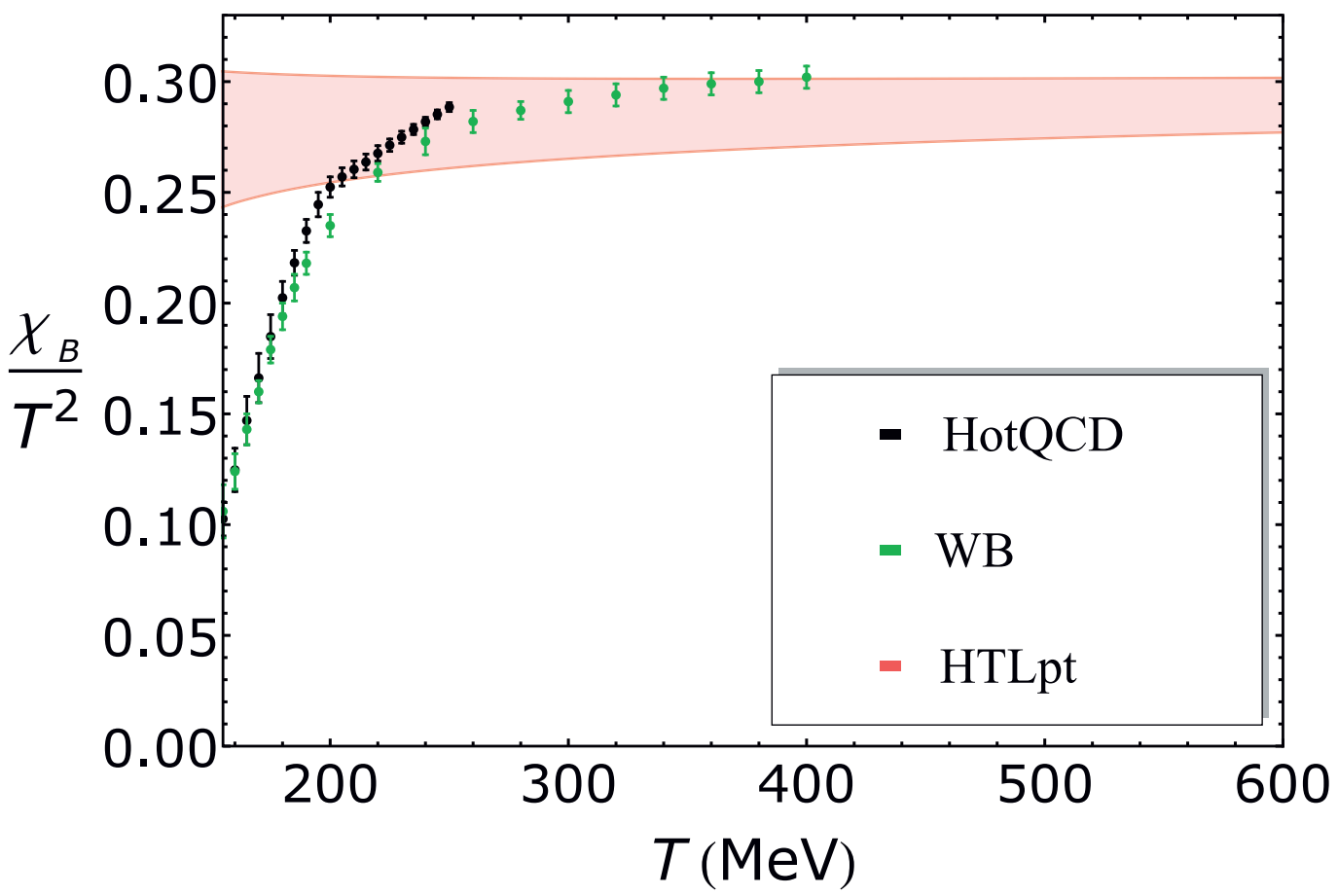

Figure 4. Comparison of the high $T$ truncation of the LO HTLpt baryon number susceptibility of $N_{f}=3$ QCD with $N_{f}=2+1$ lattice data from the HotQCD [13] and Wuppertal-Budapest [14] collaborations. Shaded band shows the result of varying the renormalization scale $\Lambda$ by a factor of 2 around $\Lambda=2 \pi T$.

\section{Outlook}

In this proceedings, we have briefly discussed how the gauge-invariant HTLpt reorganization for thermal QCD could improve the convergence of perturbation expansion at experimentally accessible temperatures, and reviewed some static results, including NNLO thermodynamic functions and LO quark/baryon number susceptibility. It has been known that the weak-coupling expansion of realtime dynamics is as poorly convergent as the thermodynamics case. Since HTLpt is formulated in Minkowski space, its application to realtime dynamics is straightforward and would be relevant for the dynamical properties of the quark gluon plasma at LHC temperatures.

\section{Acknowledgements}

The works presented in this proceedings were done in collaboration with Jens O. Andersen, Lars E. Leganger, Michael Strickland and Aleksi Vuorinen. S. M. was supported by the Sofja Kovalevskaja program and N. S. by the Postdoctoral Research Fellowship of the Alexander von Humboldt Foundation. 


\section{References}

[1] E. V. Shuryak, Sov. Phys. JETP 47, 212 (1978); S. A. Chin, Phys. Lett. B 78, 552 (1978); J. I. Kapusta, Nucl. Phys. B 148, 461 (1979); T. Toimela, Phys. Lett. B 124, 407 (1983); C. X. Zhai and B. Kastening, Phys. Rev. D 52, 7232 (1995); E. Braaten and A. Nieto, Phys. Rev. D 53, 3421 (1996); P. Ginsparg, Nucl. Phys. B 170, 388 (1980); T. Appelquist and R. D. Pisarski, Phys. Rev. D 23, 2305 (1981); K. Kajantie, M. Laine, K. Rummukainen, and M. E. Shaposhnikov, Nucl. Phys. B 458, 90 (1996); K. Kajantie, M. Laine, K. Rummukainen, and Y. Schröder, Phys. Rev. D 67, 105008 (2003); K. Kajantie, M. Laine, K. Rummukainen, and Y. Schröder, J. High Energy Phys. 04, 036 (2003).

[2] J. P. Blaizot, E. Iancu, and A. Rebhan, Phys. Rev. D 68, 025011 (2003).

[3] C. Amsler et al. [ Particle Data Group Collaboration], Phys. Lett. B 667, 1 (2008).

[4] C. McNeile et al., Phys. Rev. D 82, 034512 (2010).

[5] F. Karsch, A. Patkós, and P. Petreczky, Phys. Lett. B 401, 69 (1997).

[6] J. O. Andersen, L. E. Leganger, M. Strickland, and N. Su, Phys. Lett. B 696, (2011) 468; J. High Energy Phys. 08, (2011) 053.

[7] E. Braaten and A. Nieto, Phys. Rev. Lett. 76, 1417 (1996); Phys. Rev. D 53, 342 (1996).

[8] S. Borsanyi et al., J. High Energy Phys. 11 (2010) 077.

[9] Y. Aoki, Z. Fodor, S. D. Katz, and K. K. Szabo, J. High Energy Phys. 01 (2006) 089.

[10] A. Bazavov et al., Phys. Rev. D 80, 014504 (2009).

[11] J. O. Andersen, S. Mogliacci, N. Su, and A. Vuorinen, arXiv:1210.0912 [hep-ph].

[12] A. Bazavov et al., arXiv:1205.6155 [hep-ph].

[13] A. Bazavov et al. (HotQCD Collaboration), Phys. Rev. D 86, 034509 (2012).

[14] S. Borsanyi et al., J. High Energy Phys. 01, 138 (2012). 